\title{
A GEOMETRIC CHARACTERIZATION OF FRÉCHET SPACES WITH THE RADON-NIKODYM PROPERTY ${ }^{1}$
}

\author{
G. Y. H. CHI
}

ABSTRACT. Let $F$ be a locally convex Fréchet space. $F$ is said to have the Radon-Nikodym property if for every positive finite measure space $(\boldsymbol{\Omega}, \boldsymbol{\Sigma}, \mu)$, and every $\mu$-continuous vector measure $m: \Sigma \rightarrow F$ of bounded variation, there exists an integrable function $f: \Omega \rightarrow F$ such that $m(S)=$ $\int_{S} f(\omega) d \mu(\omega)$, for every $S \in \boldsymbol{\Sigma}$. Maynard proved that a Banach space has the Radon-Nikodym property iff it is an $s$-dentable space. It is the purpose of this paper to give the following analogous characterization: A Fréchet space $F$ has the Radon-Nikodym property iff $F$ is $s$-dentable.

0 . Introduction. In [8], Maynard obtained some equivalent geometric conditions for the average range of a vector measure in the characterization of Rieffel's Radon-Nikodym theorem [11, Main theorem, P. 466]. Based on these results, Maynard [9, Theorem 2.2] recently extended Rieffel's [12, Theorem 1] condition on the dentability of the average range to $s$-dentability of the average range. It was shown in [2], [7] that all of these results can be extended to locally convex Fréchet spaces; see $\$ 2$. As a conse. quence, the geometric characterization of Fréchet spaces having the RadonNikodym property will be proved in $\$ 3$ below.

1. Preliminaries. Let $(\Omega, \Sigma, \mu)$ be a positive finite measure space, where $\Omega$ is an abstract set, $\Sigma$ is a $\sigma$-algebra of subsets of $\Omega$, and $\mu$ is a real-valued measure defined on $\Sigma$. Without loss of generality, one can assume that $\Sigma$ is $\mu$-complete. Let $\Sigma^{+} \equiv\{S \in \Sigma \mid \mu(S)>0\}$.

Throughout this paper, let $F$ be a locally convex Fréchet space, and $\mathcal{U} \equiv\left\{U_{n}\right\}_{n=1}^{\infty}$ be a fundamental decreasing sequence of closed absolutely

Received by the editors June 14, 1973 and, in revised form, November 12, 1973.

AMS (MOS) subject classifications (1970). Primary 28A45, 46G10; Secondary 46A05.

Key words and phrases. Fréchet spaces, Radon-Nikodym property, s-dentability, dentability.

1 This was partially supported by Faculty Research Grant from the Faculty of Arts and Sciences, University of Pittsburgh. 
convex subsets which forms a 0 -neighborhood base for $F$. For $U \in \mathcal{U}$, let $P_{U}$ denote the associated continuous seminorm.

A function $f: \Omega \rightarrow F$ is said to be simple iff $f$ is of the form $f(\omega)=$ $\sum_{i=1}^{n} x_{i} I_{S_{i}}(\omega), \forall \omega \in \Omega$, where $\left\{S_{i}\right\}_{i=1}^{n} \subseteq \sum$ are disjoint and $\left\{x_{i}\right\}_{i=1}^{n} \subseteq F$. A function $f: \Omega \rightarrow F$ is said to be strongly measurable iff there exists a sequence of simple functions $\left\{f_{n}\right\}_{n=1}^{\infty}$ such that $f(\omega)=\lim _{n \rightarrow \infty} f_{n}(\omega), \forall \omega \in \Omega$. Let $\mathbb{M}(\Omega, \mu ; F)=\{f: \Omega \rightarrow F \mid f$ is strongly measurable $\}$. If $F$ is a Banach space, then $\pi(\Omega, \mu ; F)$ is precisely the Bochner measurable functions.

Let $f$ be strongly measurable, hence Borel measurable. Thus $\forall U \in \mathcal{U}$, $p_{U}(f)$ is Borel measurable. If $\forall U \in \mathcal{U}, \int_{\omega} p_{U}(f) d \mu<\infty$, then $f$ is said to be integrable. In this case define $q_{U}(f) \equiv \int_{\Omega} p_{U}(f) d \mu$. Let $\mathcal{L}^{1}(\Omega, \mu ; F)$ be the space of all integrable functions, and let $\mathcal{L}^{1}(\Omega, \mu ; F) / \Re$, where $\pi \equiv$ $\left\{f \in \mathscr{L}^{1}(\Omega, \mu ; F) \mid q_{U}(f)=0, \forall U \in \mathcal{U}\right\} . L^{1}(\Omega, \mu ; F)$ is a Fréchet space topologized by the family of seminorms $\left\{q_{U} \mid U \in \mathcal{U}\right\}$. If $F$ is a Banach space, then $L^{1}(\Omega, \mu ; F)$ is just the Banach space of Bochner integrable functions.

Let $m: \Sigma \rightarrow F$ be a vector measure. For every $U \in \mathcal{U}$, the U-variation of $m$ over $S$ is defined to be

$$
V(m, U)(S) \equiv \sup \left\{\sum_{i=1}^{n} p_{U}\left(m\left(s_{i}\right)\right) \mid s_{i} \in \Sigma, \text { disjoint, } 1 \leq i \leq n, s_{i} \subseteq s\right\} \text {. }
$$

$V(m, U)($.$) is an extended real-valued measure. m$ is said to have bounded variation if $V(m, U)(\Omega)<\infty, \forall U \in \mathcal{U} . m$ is $\mu$-continuous, denoted by $m<<$ $\mu$ if $\mu(S)=0, S \in \Sigma$, implies $m(S)=0$. It is clear that $m<<$ iff $\forall U \in \mathcal{U}$, $V(m, U)(\cdot)<<\mu$. If $f \in L^{1}(\Omega, \mu ; F)$ and $\mu_{f}(S) \equiv \int_{S} f d \mu$, then $\mu_{f}$ is a vector measure, and $\forall U \in \mathcal{U}, p_{U}\left(\mu_{f}(s)\right) \leq \int_{S} p_{U}(f) d \mu, V(\mu, U)(s)=\int_{S} p_{U}(f) d \mu$, and $\mu_{f}$ is of bounded variation.

For $S \in \Sigma^{+}$, the average range of $m$ over $S$ is defined to be the set

$$
A_{S}(m) \equiv\left\{m(T) / \mu(T) \mid T \in \Sigma^{+} \text {, and } T \subseteq S\right\} \text {. }
$$

Let $E$ be an arbitrary locally convex space, and $D \subseteq E$ be a subset. $\bar{D}$ denotes the closure of $D, \bar{D}^{\sigma}$ the $\sigma\left(E, E^{1}\right)$-closure, $c(D)$ the convex hull of $D$, and

$$
s(D) \equiv\left\{\sum_{i=1}^{\infty} \alpha_{i} d_{i} \mid \alpha_{i}>0, \sum_{i=1}^{\infty} \alpha_{i}=1, \text { and } \sum_{i=1}^{\infty} \alpha_{i} d_{i} \text { converges, } d_{i} \in D, i \geq 1\right\},
$$

the $s$-convex hull of $D$. It is clear that $D \subseteq c(D) \subseteq s(D) \subseteq c(D) . D$ is said to have width at most $U$ iff $D-D \subseteq U$, i.e., $p_{U}(x-y)<1, \forall x, y \in D$.

Definition 1.1. $D \subset E$ is said to be dentable [s-dentable] iff $\forall U \in \mathcal{U}$, there exists $d \in D$ such that $d \notin \bar{c}(D \backslash\{d+U\})[d \notin s(D \backslash\{d+U\})]$. 
If $D$ is not dentable [s-dentable], then any $U \in \mathcal{U}$ such that $\forall d \in D$, $d \in \bar{c}(D \backslash\{d+U\})[d \in s(D \backslash\{d+U\})]$ is said to be a nondenting [non-s-dent. ing] neighborhood for $D$. If $D$ is dentable, then $D$ is $s$-dentable. For the proof of the following results as well as other properties of dentability and $s$-dentability, see [2], [9], [10], [12].

Theorem 1.1. Let $F$ be a Fréchet space. Every relatively weakly com. pact subset of $F$ is dentable, and hence s-dentable.

2. Radon-Nikodym theorems. Theorem 2.1 and Theorem 2.2 below are proved in [2], [7]. For the Banach space version, Theorem 2.1 was due to Rieffel [11, Main theorem, p. 466], and Theorem 2.2 was due to Maynard [8, Theorem 3.1, p. 457].

Theorem 2.1. Let $(\Omega, \Sigma, \mu)$ be a positive finite measure space, and $F$ a Fréchet space. Let $m: \Sigma \rightarrow F$ be a vector measure. Then $m=\mu_{f}$ for some $f \in L^{1}(\Omega, \mu ; F)$ iff

(i) $m<<$,

(ii) $m$ has bounded variation,

(iii) $m$ has locally relatively compact [or s-dentable] average range, i. e., $\forall S \in \Sigma^{+}$, there exists $T \in \Sigma^{+}, T \subseteq S$ such that $A_{T}(m)$ is relatively compact [or s-dentable].

The next theorem will be used crucially in the proof of Theorem 3.1.

Theorem 2.2. Let $(\Omega, \Sigma, \mu)$ be a positive finite measure space, $F$ a Fréchet space, and $m: \Sigma \rightarrow F$ a $\mu$-continuous vector measure of bounded variation. Then the following conditions are equivalent:

(i) given $S \in \Sigma^{+}$, there exists $T \in \Sigma^{+}, T \subseteq S$ such that $A_{T}(m)$ is relatively compact.

(ii) given $S \in \Sigma^{+}$, and $U \in \mathcal{U}$, there exists $T \in \Sigma^{+}, T \subseteq S$ such that $A_{T}(m)$ has width at most $U$, i.e., $A_{T}(m)-A_{T}(m) \subseteq U$.

3. On Fréchet spaces with the Radon-Nikodym property. As in the case of Banach spaces, the concept of s-dentability provides a simple characterization of Fréchet spaces with the Radon-Nikodym property.

Definition 3.1. A Fréchet space $F$ is said to have the Radon-Nikodym Property, RNP for short, iff for any positive measure space $(\Omega, \Sigma, \mu)$ and any $m: \Sigma \rightarrow F, \mu$-continuous vector measure of bounded variation, there exists $f \in L^{1}(\Omega, \mu ; F)$ such that $m=\mu_{f}$.

The following lemma will motivate the next definition as well as the main theorem. For a proof of this lemma, see [7, Corollary 3.2]. 
Lemma 3.1. Let $(\Omega, \Sigma, \mu)$ be a positive finite measure space, and $m$ : $\Sigma \rightarrow F$ a $\mu$-continuous vector measure of bounded variation; then $m$ has locally bounded average range, i.e., $\forall S \in \Sigma^{+}$, there exists $T \in \Sigma^{+}, T \subseteq S$ such that $A_{T}(m)$ is bounded.

Definition 3.2. A Fréchet space $F$ is said to be s-dentable iff every bounded subset of $F$ is $s$-dentable.

Now the main result will be proved. The proof will be given in several stages and contains a modification of the proof presented in [9, Theorem 3.1].

Theorem 3.1. A Fréchet space $F$ has the Radon-Nikodym property iff $F$ is s-dentable.

Proof. Necessity follows from Lemma 3.1 and Theorem 2.1. To prove sufficiency, it will be shown that one can construct a positive complete finite measure space $(\Omega, \Sigma, \mu)$, and a $\mu$-continuous vector measure $m: \Sigma \rightarrow F$ of bounded variation such that $m$ has no Radon-Nikodym derivative.

Stage 1. Construction of the measurable space $(\Omega, \Sigma)$. Let $\Omega=[0,1)$ with the Euclidean topology, and let $\left\{\pi_{n}\right\}_{n=1}$ be an increasing sequence of infinite partitions of $[0,1)$ having the following properties: $\left[a_{z}^{n}, b_{z}^{n}\right)$.

(i) $\pi_{n}=\left\{A_{z}^{n}\right\}_{z \in N^{n}}$, where $N$ is the set of positive integers, and $A_{z}^{n}=$

(ii) For every $n \in N$, and every $z \in N^{n}, b_{(z, i)}^{n+1}=a_{(z, i+1)^{\circ}}^{n+1}$.

(iii) For every $n \in N$, and every $z \in N^{n}, A_{z}^{n}=\bigcup_{i=1}^{\infty} A_{(z, i)}^{n+1}$, where $(z, i) N^{n} \times N$.

Let $\pi=\bigcup_{n=1}^{\infty} \pi_{n}$, and let $R_{0}$ be the ring generated by $\pi$. It should be noted that $\pi$ is countable and is not a semiring. It is easy to see that

(1) $R_{0}=\left\{S_{1} \cup S_{2} \mid S_{1}=\bigcup_{i=1}^{n} A_{z_{i}}^{k}, S_{2}=\bigcup_{i=1}^{n}\left[\bigcup_{j=p_{i}}^{\infty} A_{\left(z_{i}, j\right)}^{{ }^{r+1}}\right], S_{1} \cap S_{2}=\varnothing\right\}$,

and that $R_{0}$ is not an algebra. Let $R$ be the algebra generated by $\pi$. It should be pointed out that neither $R_{0}$ nor $R$ contains a base for the Euclidean topology of $[0,1)$. However, $R_{0}$ does contain a base for the right half-open interval topology on $[0,1)$, which is strictly finer than the Euclidean topology $[6$, p. 37]. Let $\mathcal{D}$ be the $\delta$-ring generated by $\pi, \mathfrak{B}$ the $\delta$ ring generated by compact subsets of $[0,1)(\mathscr{B}$ is called the family of relatively compact Borel sets of $[0,1)$ in $[5, \mathrm{p} .287])$. Let

$$
\mathcal{C} \equiv\{S \subseteq[0,1) \mid S \cap B \in \mathbb{B}, \forall B \in \mathbb{B}\}
$$


$\mathcal{C}$ is a $\sigma$-algebra [5, p. 290]. One has the following inclusion relations:

$$
\pi \subseteq R_{0} \subseteq \mathfrak{D} \subseteq \mathfrak{B} \subseteq \sigma\left(R_{0}\right)=\sigma(R) \subseteq \mathcal{C}
$$

Note that $R \nsubseteq \subseteq B$, since $[0,1) \notin \Re$. The third inclusion is seen as follows. Let $[a, b) \subseteq[0,1)$. Let $a_{i}=a, a<b_{i}<b$ such that $b_{i}<b_{j}, i<j$ and $\lim _{i \rightarrow \infty} b_{i}=b$. Then $[a, b)=\bigcup_{i=1}^{\infty}\left[a_{i}, b_{i}\right]$. However, $\left[a_{i}, b_{i}\right] \in \mathcal{B},\left[a_{i}, b_{i}\right]$ $\subseteq[a, b] \in \mathcal{B}$, so by property (4) of $[5, \mathrm{p} .4],[a, b) \in \Re$. This implies that $\pi \subseteq \mathcal{B}$. But $\mathcal{B}$ is a $\delta$-ring, so $\mathscr{D} \subseteq \mathcal{B}$. To see the fourth inclusion, recall that every open subset $V \subseteq[0,1)$ has the structure

$$
V=[0, b) \cup \bigcup_{i=1}^{\infty}\left(a_{i}, b_{i}\right),
$$

where $[0, b) \in \pi,\left(a_{i}, b_{i}\right) \subset[0,1)$. However, $\forall i \geq 1,\left(a_{i}, b_{i}\right)=\bigcup_{j=1}^{\infty}\left[a_{i j}, b_{i j}\right)$, where $a_{i}<a_{i j}<b_{i}, b_{i j}=b_{i}, \forall j \geq 1$, and $\lim _{j \rightarrow \infty} a_{i j}=a_{i}$. Thus every open subset of $[0,1)$ is in $\sigma(R)$. Now if $K \subset[0,1)$ is a compact set, then $[0,1) \backslash K \in \sigma(R)$. This implies $K \in \sigma(R)$, hence $\mathfrak{B} \subset \sigma(R)$. The definition of $\Sigma$ will be given in Stage 3.

Stage 2. Construction of $\mu$ and $m$ on $R_{0}$. Let $D \subset F$ be a nonempty bounded not $s$-dentable set. There exists then $U_{0} \in \mathcal{U}$, and $\alpha_{0}>0$ such that

(a) $U_{0}$ is a non-s-denting neighborhood for $D$, i.e., $\forall d \in D, d \in$ $s\left(D \backslash\left\{d+U_{0}\right\}\right)$, and

(b) $D \subset a_{0} U_{0}$.

One defines $\mu$ and $m$ inductively on $\pi$ as follows:

(i) Let $d_{0} \in D$ be arbitrary. By (a), there exists $\alpha_{i}^{1}>0, i \geq 1$, $\sum_{i=1}^{\infty} \alpha_{i}^{1}=1$, and $\left\{d_{i}^{1}\right\}_{i=1}^{\infty} \subseteq D \backslash\left\{d_{0}+U_{0}\right\}$ such that $d_{0}=\sum_{i=1}^{\infty} \alpha_{i}^{1} d_{i}^{1}$. On $\pi_{1}$, define $\mu\left(A_{i}^{1}\right)=\alpha_{i}^{1}$, and $m\left(A_{i}^{1}\right)=\alpha_{i}^{1} d_{i}^{1}, i \geq 1$.

(ii) Now suppose that $\mu$ and $m$ have been defined on $\pi_{n}$ such that $\forall n \in N$, and $\forall z \in N^{n}, m\left(A_{z}^{n}\right) / \mu\left(A_{z}^{n}\right)=d_{z}^{n} \in D, \forall A_{z}^{n} \in \pi_{n}$. Since $D$ is not $s$-dentable, there exists $\alpha_{(z, i)}^{n+1}>0, \sum_{i=1}^{\infty} \alpha_{(z, i)}^{n+1}=1$, and $\left\{d_{(z, i)}^{n+1}\right\}_{i=1}^{\infty} \subseteq$ $D \backslash\left\{d_{z}^{n}+U_{0}\right\}$ such that $d_{z}^{n}=\sum_{i=1}^{\infty} \alpha_{(z, i)}^{n+1} d_{(z, i)}^{n+1}$. Define

$$
\mu\left(A_{(z, i)}^{n+1}\right) \equiv \alpha_{(z, i)}^{n+1} \text { and } \quad m\left(A_{(z, i)}^{n+1}\right) \equiv \alpha_{(z, i)}^{n+1} d_{(z, i)^{\circ}}^{n+1}
$$

Observe that

$$
m\left(A_{(z, i)}^{n+1}\right) / \mu\left(A_{(z, i)}^{n+1}\right)-m\left(A_{z}^{n}\right) / \mu\left(A_{z}^{n}\right) \notin U_{0}
$$

By induction, $\mu$ and $m$ are thus defined on all of $\pi$ satisfying the crucial "horizontal" countable additivity property: $\forall A_{z}^{n} \in \pi$, 


$$
\mu\left(A_{z}^{n}\right)=\sum_{i=1}^{\infty} \mu\left(A_{(z, i)}^{n+1}\right) \text { and } m\left(A_{z}^{n}\right)=\sum_{i=1}^{\infty} m\left(A_{(z, i)}^{n+1}\right)
$$

Now $\mu$ and $m$ will be defined on $R_{0}$ as follows: Let

$$
S_{1}=\bigcup_{i=1}^{n} A_{z_{i}}^{k_{i}} \text { and } S_{2}=\bigcup_{i=1}^{n_{2}} \bigcup_{j=p_{i}}^{\infty} A_{\left(z_{i}, j\right)}^{r_{i}^{+1}}
$$

such that $S_{1} \cap S_{2}=\varnothing$. Define

$$
\mu\left(S_{1}\right) \equiv \sum_{i=1}^{n_{1}} \mu\left(A_{z_{i}}^{k_{i}}\right) \text { and } m\left(S_{1}\right) \equiv \sum_{i=1}^{n_{1}} m\left(A_{z_{i}}^{k_{i}}\right)
$$

Using (6) and (7), one can define

$$
\mu\left(S_{2}\right)=\sum_{i=1}^{n_{2}}\left[\mu\left(A_{z_{i}}^{r_{i}}\right)-\sum_{j=1}^{p_{i}-1} \mu\left(A_{\left(z_{i}, j\right)}^{r_{i+1}}\right)\right] \text {, and }
$$

$$
m\left(S_{2}\right) \equiv \sum_{i=1}^{n}\left[m\left(A_{z_{i}}^{r_{i}}\right)-\sum_{j=1}^{p_{i}-1} m\left(A_{\left(z_{i}, j\right)}^{r_{i}+1}\right)\right] .
$$

Finally define $\mu\left(S_{1} \cup S_{2}\right) \equiv \mu\left(S_{1}\right)+\mu\left(S_{2}\right)$, and $m\left(S_{1} \cup S_{2}\right) \equiv m\left(S_{1}\right)+m\left(S_{2}\right)$ where the right sides of the above equations are defined by (7) and (8).

It is easy to check that $\mu$ and $m$ are finitely additive on $R_{0}$, and that $\mu$ is a positive set function of bounded variation. In fact, the variation of $\mu$ is bounded by 1 . Furthermore, by first proving for sets of $\pi$ then for sets of $R_{0}$ by using the definition of $m$ on $\pi$ and the structure of $R_{0},(1)$, one can show that $\forall U \in \mathcal{U}$, there exists $\alpha_{U}>0$ such that

$$
p_{U}(m(A))<\alpha_{U} \mu(A), \quad \forall A \in R_{0} .
$$

Stage 3. Extension of $\mu$ and $m$. Using the "horizontal" countable additivity property (6) and the structure of $\pi_{n}$, one can prove that $\mu$ is regular on $\pi$ relative to $R_{0}$, i.e., $\forall A \in \pi$, and $\forall \epsilon>0$, there exist $C, V \in R_{0}$ such that $C \subseteq A \subseteq V^{0}$, and $\mu(H)<\epsilon, \forall H \in \Re_{0}, H \subseteq V \backslash C$ (see [9, p. 11]). Then knowing the structure of $\mathcal{R}_{0}$, and (1), one can prove by direct computation that $\mu$ is regular on $R_{0}$ relative to $R_{0}$ (this was termed regular $R_{2}$ in [4, p. 508]). Hence by Theorem 3 of [4, p. 510], $\mu$ is countably additive on $R_{0}$. By the Carathéodory extension procedure, $\mu$ can be extended to a unique finite positive measure $\mu_{1}$ on $\mathcal{C}(\mu)$, the class of $\mu$-measurable sets [5, Corollary 4, Proposition 6, p. 72, Definition 3, p. 67]. Since $\mu_{1}$ is finite, $\Sigma(\mu)$, the class of $\mu$-integrable sets coincide in this case with $\mathcal{C}(\mu)$, and $\mu_{1}$ 
is a complete measure on $\Sigma(\mu)$ [5, Proposition 12, Definition 6, p. 75]. Furthermore, $R_{0}$ is $\mu_{1}$-dense in $\Sigma(\mu)$, i.e., $\forall S \in \Sigma(\mu)$, and $\forall \epsilon>0$, there exists $B \in R_{0}$ such that $\mu_{1}(S \Delta B)<\epsilon[5$, Proposition 13, p. 76]. It should be emphasized that the extension procedure need not preserve regularity! Hence $\mu_{1}$ need not be regular on $\Sigma(\mu)$, in fact, $\mu_{1}$ need not even be regular on $\sigma(R)$, in the sense that $\forall S \in \sigma(\Re)$ and $\forall \epsilon>0$, there exists $C, V \subseteq[0,1)$ such that $\bar{C} \subseteq S \subseteq V^{0}$, and $\mu(H)<\epsilon, \forall H \in \sigma(R), H \subseteq V \backslash C$, without the requirement that $C, V \in \sigma(\Re)$.

Let $\Sigma(\mu)$ be denoted by $\Sigma$, and $\mu_{1}$ again by $\mu$. Since $\mu$ is countably additive, (9) and Lemma 1 of $[4$, p. 506] implies that $m$ is countably additive on $R_{0}$. Since $F$ is complete, $\mu$ is countable additive, and $R_{0}$ is $\mu$ dense in $\Sigma$, one can prove (analogous to the proof of Theorem 1, p. 62 of [5]) that $m$ can be extended to a measure $m_{1}: \Sigma \rightarrow F$ such that $\forall U \in \mathcal{U}$, there exists $\alpha_{U}>0$ such that

$$
P_{U}\left(m_{1}(S)\right) \leq \alpha_{U} \mu(S), \quad \forall S \in \Sigma .
$$

Since $\mu$ is of bounded variation on $\Sigma$, it follows from (10) that $m_{1}$ has bounded variation on $\Sigma$. Again $m_{1}$ need not be regular on $\Sigma$, or on $\sigma(R)$. Let $m_{1}$ be denoted by $m$.

The restriction of $\mu$ to $\mathcal{B}$ is a positive finite measure, so by Corollary 2 of $[5$, p. 347], $\mu: 3 \rightarrow[0,1]$ is a regular Borel measure (i.e., $\forall B \in \mathfrak{B}$ and $\epsilon>0$, there exists $C, V \subseteq[0,1)$ such that $\bar{C} \subseteq B \subseteq V^{0}$, and $\mu(H)<\epsilon, \forall$ $H \in \mathfrak{B}, H \subseteq V \backslash C)$. Now from (10) and Lemma 3 of [4, p. 509], $m: \mathfrak{B} \rightarrow F$ is a regular Borel measure of bounded variation. However, the regularity of $m$ on $\mathfrak{B}$ will not be needed.

Thus one has constructed a measure space $(\Omega, \Sigma, \mu)$ and a $\mu$-continuous vector measure $m: \Sigma \rightarrow F$ of bounded variation such that the restrictions of $\mu$ and $m$ to $\mathfrak{B}$ are regular Borel measures.

Stage 4. $m \neq \mu_{f}$, for any $f \in L^{1}(\Omega, \mu ; F)$. It suffices to show that $\forall B \in$ $\mathfrak{B}^{+}, A_{B}(m)$ has width at least $1 / 2 U_{0}$. (In fact, it should be pointed out that it is not necessarily true that every $S \in \sigma(R)^{+}, A_{S}(m)$ has width at least $\left.1 / 2 U_{0^{\circ}}\right)$

Let $B \in \mathfrak{B}^{+}$. By regularity of $\mu$ on $\mathfrak{B}$, one can find a compact $C$ and an open $V$ such that

(i) $\bar{C} \subseteq B \subseteq V^{0}$,

(ii) $\mu(\bar{H})<\left(1 / 16 a_{0}\right) \mu(B), \forall H \in \mathfrak{B}, H \subseteq V \backslash C$, where $D \subseteq a_{0} U_{0}$.

Now by the third and fourth inclusions in (3) and Proposition 7 of [5, p. 73], one has

(iii) $\mu(V \backslash C) \leq\left(1 / 16 \alpha_{0}\right) \mu(B)$, where $D \subseteq \alpha_{0} U_{0}$. 
Now by the structure of $V$, and (4), there exists $\left\{A_{i}\right\}_{i=1}^{\infty} \subset \pi$ such that (iv) $C \subseteq \bigcup_{i=1}^{\infty} A_{i} \subseteq V$.

There exists at least one $A_{i_{0}}$ such that

$$
\mu\left(A_{i_{0}} \backslash B\right) / \mu\left(A_{i_{0}}\right) \leq 1 / 8 \alpha_{0} .
$$

Let $G=A_{i_{0}} \cap B \in \mathbb{B}$. Then $G \subseteq B$, and $\mu(G)>0$. Since $A_{i_{0}} \in \pi$, by taking the next partition of $A_{i_{0}}$, there exists disjoint $\left\{C_{k}\right\}_{k=1}^{\infty}$ such that $A_{i_{0}}=$ $\bigcup_{k=1}^{\infty} C_{k}$. Claim that there exists $C_{n}$ such that

$$
\mu\left(C_{n} \backslash B\right)<\left(1 / 8 a_{0}\right) \mu\left(C_{n}\right) .
$$

For otherwise,

$$
\mu\left(A_{i_{0}} \backslash B\right)=\mu\left(\bigcup_{k=1}^{\infty}\left(C_{k} \backslash B\right)\right)>\frac{1}{8} \frac{1}{a_{0}} \sum_{k=1}^{\infty} \mu\left(C_{k}\right)=\frac{1}{8 a_{0}} \mu\left(A_{i_{0}}\right)
$$

which contradicts (11).

Let $H=C_{n} \cap B \in$ B. Since $C_{k}$ belongs to the next partition of $A_{i_{0}}$, by (5),

$$
P_{U_{0}}\left(m\left(A_{i_{0}}\right) / \mu\left(A_{i_{0}}\right)-m\left(C_{n}\right) / \mu\left(C_{n}\right)\right)>1
$$

Direct computation shows that

$$
P_{U_{0}}\left(\frac{m(G)}{\mu(G)}-\frac{m\left(A_{i_{0}}\right)}{\mu\left(A_{i_{0}}\right)}\right)<\frac{1}{4} \quad \text { and } \quad P_{U_{0}}\left(\frac{m(H)}{\mu(H)}-\frac{m\left(C_{n}\right)}{\mu\left(C_{n}\right)}\right)<\frac{1}{4} \text {. }
$$

Thus,

$$
\begin{aligned}
& P_{U_{0}}\left(\frac{m(G)}{\mu(G)}-\frac{m(H)}{\mu(H)}\right) \geq P_{U_{0}}\left(\frac{m\left(A_{i_{0}}\right)}{\mu\left(A_{i_{0}}\right)}-\frac{m\left(C_{n}\right)}{\mu\left(C_{n}\right)}\right) \\
&-P_{U_{0}}\left(\frac{m\left(A_{i_{0}}\right)}{\mu\left(A_{i_{0}}\right)}-\frac{m(G)}{\mu(G)}\right)-P_{U_{0}}\left(\frac{m(H)}{\mu(H)}-\frac{m\left(C_{n}\right)}{\mu\left(C_{n}\right)}\right) \\
&> 1-1 / 4-1 / 4 \text { (by (13) and (14)) } \\
&> 1 / 2 .
\end{aligned}
$$

Thus,

$$
m(G) / \mu(G)-m(H) / \mu(H) \notin 1 / 2 U_{0} .
$$

Thus $\forall B \in B^{+}, A_{B}(m)$ has width at least $1 / 2 U_{0}$, so by Theorem 2.1 
and Theorem 2.2, $m \neq \mu_{f}$, for any $f \in L^{1}(\Omega, \mu ; F)$. For if there exists such an $f$, then by Theorem 2.1 and Theorem 2.2, $\forall S \in \Sigma^{+}, \forall U \in \mathcal{U}$, there exists $T \in \Sigma^{+}, T \subseteq S$ such that $A_{T}(m)$ has width at most $U$. This should hold in particular for every $B \in \mathfrak{B}^{+} \subseteq \sigma(R)^{+}$. In view of (15), this is not possible for $U=1 / 2 U_{0}$. For suppose there exists $T \in \sigma(R)^{+}, T \subseteq B$, such that $A_{T}(m)$ has width at most $1 / 2 U_{0}$. But by the definition of $\mathcal{C},(2)$, and the last inclusion relation in (3), $T \in B^{+}$, and hence by (15), $A_{T}(m)$ has width at least $1 / 2 U_{0}$, hence a contradiction. Q.E.D.

As a corollary, one deduces the following result of [7].

Corollary 3.1. Every reflexive Fréchet space $F$ has the RNP.

Proof. If $F$ is a reflexive Fréchet space, then by Theorem 5.6 of $[11$, p. 145], every bounded set $D \subseteq F$ is relatively weakly compact. By Theorem $1.1, D$ is s-dentable, and so by Theorem 3.1, $F$ has RNP. Q.E.D.

Remark 3.1. The above corollary holds, in particular, if $F$ is a nuclear Fréchet space, or separable dual of a barreled $(D F)$-space, or Fréchet-Montel space.

Remark 3.2. Corollary 3.1 shows that in a reflexive Fréchet space, every bounded set is dentable. Whether this remains true if $F$ is s-dentable is still an open question. A related question is whether $F$ has RNP iff $F$ is dentable. These are known to be true for Banach spaces.

\section{REFERENCES}

1. G. Y. H. Chi, The Radon-Nikodym theorem for vector measures with values in the duals of some nuclear barreled spaces, Proc. Sympos。 on Vector and Operator Valued Measures and Applications, Salt Lake City, Utah, August, 1972, Academic Press, New York, 1973, pp. 85-95.

2. - The Radon-Nikodym theorems for Fréchet spaces (preprint).

3. N. Dinculeanu, On regular vector measures, Acta Sci. Math. (Szeged) 24 (1963), 236-243. MR $31 \# 300$.

4. N. Dinculeanu and I. Kluvanek, On vector measures, Proc. London Math. Soc. (3) 17 (1967), 505-512. MR $35 \# 5571$.

5. N. Dinculeanu, Vector measures, Internat. Series of Monographs in Pure and Appl. Math., vol 95, Pergamon Press, Oxford; VEB Deutscher Verlag der Wissenschaften, Berlin, 1967. MR 34 \#6011b.

6. S. A. Gaal, Point set topology, Pure and Appl. Math., vol. 16, Academic Press, New York, 1964. MR 30 \#1484.

7. D. R. Lewis, On the Radon-Nikodym theorem (preprint).

8. H. B. Maynard, A Radon-Nikodym theorem for operator-valued measures, Trans. Amer. Math. Soc. 173 (1972), 449-463.

9. - A geometric characterization of Banach spaces having the Radon- 
Nikodym property (preprint); cf., Trans. Amer. Math. Soc. 173 (1972), 449-463.

10. I. Namioka, Neighborhoods of extreme points, Israel J. Math. 5 (1967), 145152. MR $36 \# 4323$.

11. M. A. Rieffel, The Radon Nikodym theorem for the Bochner integral, Trans. Amer. Math. Soc. 131 (1968), 466-487。 MR 36 \#5297.

12. - Dentable subsets of Banach spaces, with application to a Radon-Nikodým theorem, Functional Analysis (Proc. Confo, Irvine, Calif., 1966), Academic Press, London; Thompson Book Co., Washington, D。C., 1967, pp. 71-77. MR $36 \# 5668$ 。

13. H. H. Schaefer, Topological vector spaces, Macmillan, New York, 1966. MR $33 \# 1689$.

DEPARTMENT OF MATHEMATICS, UNIVERSITY OF PITTSBURGH, PITTSBURGH, PENNSYLVANIA 15260 